\title{
Quantum control and the Strocchi map
}

\author{
R. Vilela Mendes* \\ Complexo Interdisciplinar, Universidade de Lisboa \\ Av. Prof. Gama Pinto, 2, 1699 Lisboa Codex, Portugal \\ V. I. Man'ko \\ P. N. Lebedev Physical Institute \\ Leninsky Prospect 53, 117924 Moscow, Russia
}

\begin{abstract}
Identifying the real and imaginary parts of wave functions with coordinates and momenta, quantum evolution may be mapped onto a classical Hamiltonian system. In addition to the symplectic form, quantum mechanics also has a positive-definite real inner product which provides a geometrical interpretation of the measurement process. Together they endow the quantum Hilbert space with the structure of a Käller manifold.

Quantum control is discussed in this setting. Quantum timeevolution corresponds to smooth Hamiltonian dynamics and measurements to jumps in the phase space. This adds additional power to quantum control, non unitarily controllable systems becoming controllable by "measurement plus evolution".

A picture of quantum evolution as Hamiltonian dynamics in a classical-like phase-space is the appropriate setting to carry over techniques from classical to quantum control. This is illustrated by a discussion of optimal control and sliding mode techniques.
\end{abstract}

*corresponding author: vilela@cii.fc.ul.pt 


\section{Introduction}

The mathematical structures of classical and quantum mechanics are usually regarded as essentially different. However, many years ago Strocchi[1], by identifying the real and imaginary parts of the wave function with coordinates and momenta, has shown that quantum evolution may be mapped onto a classical Hamiltonian system. In particular this setting suits nicely a geometrical interpretation of quantum mechanics. This formulation of quantum mechanics, first proposed by Strocchi[1], has since been rediscovered and extended by many authors[2] [3] [4] [5] [6]. Some applications of the Strocchi map to the evolution of finite-dimensional quantum systems were considered in [7] and structures relevant to the relation of classical to quantum mechanics were studied in [8]. A particularly interesting extension to the original ideas of the Strocchi map was the realization that, in addition to the symplectic form, characteristic of Hamiltonian evolution, quantum mechanics also has a positive-definite real inner product. Together they endow the quantum Hilbert space with the structure of a Käller manifold[3] [6]. We will discuss this more general framework of the Strocchi map. However, for simplicity and whenever possible, we will adhere to the original intuitive coordinate formulation of Strocchi.

The aim of this work is to discuss quantum control in the geometrical setting provided by the Strocchi map. Quantum time-evolution will correspond to smooth Hamiltonian dynamics in a classical-like phase-space and measurements to jumps in the phase-space. This is very different from the situation in classical feedback control, where the measurements needed for the feedback action are not supposed to change the state of the system or to change it only very little. However, in some cases, rather than being a nuisance, the state disturbance introduced by quantum measurement adds additional power to quantum feedback control and, in particular, it changes the question of controllability. Non unitarily controllable systems may become controllable by "measurement plus evolution".

The paper is organized as follows: In section 2 a review is made of the properties of the Strocchi map as well as some extensions needed to describe quantum evolution and control in both the von Neumann and positiveoperator-valued measure approaches. In section 3, a new quantum control method is proposed which combines free quantum evolution and quantum measurement to reach a desirable quantum state. Finally, in section 4, quan- 
tum analogs of classical nonlinear and optimal control methods are shortly discussed.

\section{Geometrical formulation of quantum me- chanics. The Strocchi map}

Consider a basis $\{|k\rangle\}$ in a separable complex Hilbert space $\mathcal{H}^{*}$. A general quantum state $|\psi\rangle$ is

$$
|\psi\rangle=\sum_{k} \psi_{k}|k\rangle
$$

Define

$$
\psi_{k}=\frac{1}{\sqrt{2}}\left(q_{k}+i p_{k}\right)
$$

where $\left\{q_{k}, p_{k}\right\}$ is a numerable set of real phase-space coordinates. Then the scalar product in the complex Hilbert space $\mathcal{H}^{*}$

$$
\left\langle\psi^{\prime} \mid \psi\right\rangle=\frac{1}{2} \sum_{k}\left(q_{k}^{\prime} q_{k}+p_{k}^{\prime} p_{k}\right)+i\left(q_{k}^{\prime} p_{k}-p_{k}^{\prime} q_{k}\right)=\frac{1}{2}\left\{G\left(\psi^{\prime}, \psi\right)+i \Omega\left(\psi^{\prime}, \psi\right)\right\}
$$

decomposes into the sum of a positive real inner product

$$
G\left(\psi^{\prime}, \psi\right)=\frac{1}{2} \sum_{k}\left(q_{k}^{\prime} q_{k}+p_{k}^{\prime} p_{k}\right)
$$

and a symplectic form

$$
\Omega\left(\psi^{\prime}, \psi\right)=\frac{1}{2} \sum_{k}\left(q_{k}^{\prime} p_{k}-p_{k}^{\prime} q_{k}\right)
$$

Considering $\mathcal{H}^{*}=(\mathcal{H}, J)$ as a real Hilbert space $\mathcal{H}$ with a complex structure $J$, the triple $(J, G, \Omega)$ equips $\mathcal{H}$ with the structure of a Kähler space because

$$
G\left(\psi^{\prime}, \psi\right)=\Omega\left(\psi^{\prime}, J \psi\right)
$$

The Schrödinger equation $i \frac{\partial}{\partial t}|\psi\rangle=H|\psi\rangle$ becomes the set of Hamilton's equations

$$
\begin{aligned}
\frac{d}{d t} q_{k} & =\frac{\partial}{\partial p_{k}} \mathbb{H} \\
\frac{d}{d t} p_{k} & =-\frac{\partial}{\partial q_{k}} \mathbb{H}
\end{aligned}
$$


associated to the symplectic form $\Omega\left(\psi^{\prime}, \psi\right)$ and the "classical" Hamiltonian

$$
\mathbb{H}=\frac{1}{2} \sum_{k, j}\left\{\left(q_{k} q_{j}+p_{k} p_{j}\right) \operatorname{Re} H_{k j}+\left(p_{k} q_{j}-q_{k} p_{j}\right) \operatorname{Im} H_{k j}\right\}
$$

with $H_{k j}=\langle k|H| j\rangle$.

One sees that the time evolution of quantum mechanics is equivalent to the classical dynamics of a numerable set of coupled oscillators. What is unique to quantum mechanics is the special role played by the symmetric form $G\left(\psi^{\prime}, \psi\right)$.

Let $\mathcal{S}$ be the Hilbert sphere, that is, the space of normalized $(\|\psi\|=1)$ functions in the Hilbert space $\mathcal{H} . G\left(\psi^{\prime}, \psi\right)$ defines a metric in $\mathcal{S}$. Consider now a measurement of an observable $A$ which, for simplicity, we assume to have a (possibly degenerate) discrete spectrum. Let $a$ be a (degenerate) eigenvalue of $A$ and $P_{a}$ the projector on the subspace $V_{a}$ of $\mathcal{S}$ associated to this eigenvalue. After the measurement of $A$ is performed and the value is found to be $a$, the quantum state changes from $\psi \in \mathcal{S}$ to $\psi_{a}=\frac{P_{a} \psi}{\left\|P_{a} \psi\right\|} \in \mathcal{S}$ and the probability to find this value is $\left\|P_{a} \psi\right\|^{2}$. The metric $G\left(\psi^{\prime}, \psi\right)$ provides a nice geometrical interpretation of the measurement process in quantum mechanics.

Given $\psi \in \mathcal{S}$ and $\phi \in V_{a} \subset \mathcal{S}$ it is easy to see that

$$
(\psi-\phi, \psi-\phi)
$$

is minimal when $\phi=\psi_{a}$. Because $(\psi-\phi, \psi-\phi)=G(\psi-\phi, \psi-\phi)$ one concludes that the measurement projects $\psi$ on the element of $V_{a}$ that is closest to $\psi$ in the $G$-metric. The probability for this projection is

$$
p_{a}=\left\|P_{a} \psi\right\|^{2}=\left(1-\frac{1}{2} G\left(\psi-\frac{P_{a} \psi}{\left\|P_{a} \psi\right\|}, \psi-\frac{P_{a} \psi}{\left\|P_{a} \psi\right\|}\right)\right)^{2}
$$

Therefore, whereas the symplectic form $\Omega$ determines time-evolution, the $G$-metric controls the measurement process. It is the special role played by the metric that, in this framework, sets apart quantum from classical mechanics.

In the numerable basis $\{|k\rangle\}$ of finite or infinite cardinality $\chi$, each pure quantum state is represented by a point $(\vec{q}, \vec{p})$ in a "phase-space" of dimension $2 \chi$. Similarly a mixed state will be described by a density 
on the same space. The density matrix for a mixed state of the form $\rho(t)=\sum_{n} \rho_{n}\left|\psi_{n}(t)\right\rangle\left\langle\psi_{n}(t)\right|\left(\sum_{n} \rho_{n}=1\right)$ becomes, using the notations in Eq. (2)

$$
\rho(t)=\int d \vec{q} d \vec{p} \rho(\vec{q}, \vec{p}) \sum_{k, k^{\prime}}\left(q_{k}(t)+i p_{k}(t)\right)\left(q_{k^{\prime}}(t)-i p_{k^{\prime}}(t)\right)|k\rangle\left\langle k^{\prime}\right|
$$

Using the equations of motion (7) and integration by parts one obtains the following classical-like equation for the "phase-space" density

$$
\frac{d}{d t} \rho(\vec{q}, \vec{p})=-\frac{\partial \rho}{\partial \vec{q}} \cdot \frac{\partial \mathbb{H}}{\partial \vec{p}}+\frac{\partial \rho}{\partial \vec{p}} \cdot \frac{\partial \mathbb{H}}{\partial \vec{q}}=-\{\rho, \mathbb{H}\}
$$

In the Strocchi map framework, the (unobserved) dynamics of quantum states is a continuous symplectic evolution in a phase space. On the other hand, measurement of a state is represented by jumps in phase space. Because the basis $\{|k\rangle\}$ is arbitrary, we may suppose that this is a basis of eigenstates of the set $\mathcal{K}$ of observables that is being measured. Let the state before the measurement be $(\vec{q}, \vec{p})$. When a measurement is performed and the results registered to be $k$, the state jumps from $(\vec{q}, \vec{p})$ to $\left(\vec{q}=\frac{q_{k}}{\sqrt{q^{2}+p^{2}}} \overrightarrow{e_{k}}, \vec{p}=\frac{p_{k}}{\sqrt{q^{2}+p^{2}}} \overrightarrow{e_{k}^{\prime}}\right)$ where $\overrightarrow{e_{k}}$ and $\overrightarrow{e_{k}}$ are unit vectors along the $k$-coordinate and the $k$-momentum.

In feedback control, the results of a measurement are used to correct the driving forces and different corrections will be associated to different results of the measurement. Therefore the kind of measurement of interest in quantum control is a selective one, that is, one in which the result of the measurement is registered. However, in quantum mechanics, even if the results of the measurement are no registered, the state of the system (or our information about it) is changed anyway. For such non-selective measurements one obtains a mixed state. If, for example the initial state is a normalized pure state, corresponding to the phase space vector $(\vec{q}, \vec{p})$, after the measurement the state corresponds to a phase-space density

$$
\rho(\vec{\mu}, \vec{\nu})=\sum_{k}\left(q_{k}^{2}+p_{k}^{2}\right) \delta\left(\vec{\mu}-\frac{q_{k}}{\sqrt{q^{2}+p^{2}}} \overrightarrow{e_{k}}\right) \delta\left(\vec{\nu}-\frac{p_{k}}{\sqrt{q^{2}+p^{2}}} \overrightarrow{e_{k}}\right)
$$


The above considerations refer to complete quantum mechanical projections, that is to (selective or non-selective) outputs of quantum mechanical measurements. A description of the behavior of a quantum system under continuous observation also exists. It uses generalized quantum measurements implemented as positive operator-valued measures. Caves and Milburn[9] assume that any measurement takes a certain amount of time and that, in the infinitesimal time interval $d t$, the measurement of the observable $\Lambda$ corresponds to the operation

$$
\rho \rightarrow P_{\Lambda} \rho P_{\Lambda}
$$

with

$$
P_{\Lambda}(\alpha)=\left(\frac{\pi}{2 s d t}\right)^{-\frac{1}{4}} e^{-s d t(\Lambda-\alpha)^{2}}
$$

and $\int d \alpha P_{\Lambda}^{\dagger}(\alpha) P_{\Lambda}(\alpha)=1$. Applying $P_{\Lambda}(\alpha)$ to any state one obtains a superposition of eigenstates of $\Lambda$ with eigenvectors centered around $\alpha$ and $s$ defines the resolution (or the strength) of the measurement. Notice however, that $P_{\Lambda}(\alpha)$ is not a projection. It is supposed [9] to represent a generalized selective measurement. For a non-selective measurement, that is, one where the results are not recorded,

$$
\rho \rightarrow \int d \alpha P_{\Lambda}(\alpha) \rho P_{\Lambda}(\alpha)
$$

From Eqs.(15)-(16) and the unconditional evolution $\frac{d \rho}{d t}=-i[H, \rho]$, the following non-selective continuous observation equation for the density matrix is obtained

$$
\frac{d \rho}{d t}=-i[H, \rho]-\frac{s}{2}[\Lambda,[\Lambda, \rho]]
$$

This equation is physically appealing, in the sense that, for example, the last double commutator term displays a mechanism for the damping of nondiagonal terms in the density matrix. Notice however that the $P_{\Lambda}(\alpha)$ operations are quantum mechanical projections only in the limit of infinite strength, or infinite time.

Because the choice of basis is arbitrary we may, without loss of generality, choose a basis of eigenstates of the measured observable $\Lambda$. Then, one has the following equation for the matrix elements of the density matrix

$$
\frac{d}{d t} \rho_{k k^{\prime}}=-i\left\langle k|[H, \rho]| k^{\prime}\right\rangle-\frac{s}{2}\left(\lambda_{k}-\lambda_{k^{\prime}}\right)^{2} \rho_{k k^{\prime}}
$$


leading to the damping of non-diagonal terms, $\lambda_{k}$ and $\lambda_{k^{\prime}}$ being the eigenvalues of $\Lambda$ in the states $|k\rangle$ and $\left|k^{\prime}\right\rangle$.

From the Strocchi map "phase-space" density point of view, Eq.(18) means that, if the initial state is a pure state $\rho_{0}(\vec{\mu}, \vec{\nu})=\delta(\vec{\mu}-\vec{q}) \delta(\vec{\nu}-\vec{p})$, (in addition to the Hamiltonian evolution) continuous observation splits the density into several components which, when $t \rightarrow \infty$, converge to a density as in (13). Independently of the conceptual interest of generalized measurements and continuous observation, the important point to retain is that the operation of measurement modifies Hamiltonian evolution. Hence, it might play for quantum control a role similar to the one that is played by dissipation in non-linear classical control techniques.

\section{Control by measurement plus evolution}

As seen before, in the Strocchi map phase-space, undisturbed time-evolution is a smooth Hamiltonian dynamics in phase-space, whereas measurements correspond to jumps in the phase-space. This last aspect is very different from the situation in classical feedback control, where the measurements needed for the feedback action are not supposed to change the state of the system or to change it only very little. However in some cases, rather than being a nuisance, the state disturbance introduced by quantum measurement adds additional power to quantum feedback control. In particular it changes the question of controllability.

For quantum systems with Hamiltonian

$$
H(t)=H_{0}+\sum_{j=1}^{r} u_{j}(t) H_{j}
$$

Huang, Tarn and Clark[10] obtained a general result on controllability involving the Lie algebras generated by the free and control Hamiltonians. A bounded quantum system with finite energy has a finite number $N$ of allowed states. For this case a necessary and sufficient condition for controllability is that the Lie algebra generated by $\left\{H_{0}, H_{1}, \cdots, H_{r}\right\}$ be $u(N)[12]$ (or $s u(N)$ if phases are not important[11]) because this is the smallest group that acts transitively on the complex sphere $S_{\mathbb{C}}^{N-1}$.

When the Strocchi map is used to describe quantum evolution of the $N$-level system, the phase-space has dimension $2 N$ with coordinates $\left\{q_{k}, p_{k}\right\}$. 
The set of transformations that need to be available to have controllability is the $U(N)$ subgroup of $S O(2 N)$ corresponding to the real linear canonical transformations, that also preserve the form $G\left(\psi^{\prime}, \psi\right)$ (Eq.(4)). It contains $N(N-1) / 2$ one-parameter subgroups of the type

$$
\begin{aligned}
q_{i}^{\prime} & =q_{i} \cos \theta+q_{j} \sin \theta \\
q_{j}^{\prime} & =-q_{i} \sin \theta+q_{j} \cos \theta \\
p_{i}^{\prime} & =p_{i} \cos \theta+p_{j} \sin \theta \\
p_{j}^{\prime} & =-p_{i} \sin \theta+p_{j} \cos \theta
\end{aligned}
$$

$N(N-1) / 2$ of type

$$
\begin{aligned}
q_{i}^{\prime} & =q_{i} \cos \theta-p_{j} \sin \theta \\
q_{j}^{\prime} & =-p_{i} \sin \theta+q_{j} \cos \theta \\
p_{i}^{\prime} & =p_{i} \cos \theta+q_{j} \sin \theta \\
p_{j}^{\prime} & =q_{i} \sin \theta+p_{j} \cos \theta
\end{aligned}
$$

and $N$ of type

$$
\begin{aligned}
& q_{i}^{\prime}=q_{i} \cos \theta-p_{i} \sin \theta \\
& p_{i}^{\prime}=q_{i} \sin \theta+p_{i} \cos \theta
\end{aligned}
$$

Suppose now that $\mathcal{A}=\left\{H_{0}, H_{1}, \cdots, H_{r}\right\}_{L A}$ is a proper subalgebra of $u(N)$. Then each orbit of the subgroup $G(\mathcal{A}) \subset U(N)$ does not cover $S_{\mathbb{C}}^{N-1} . S_{\mathbb{C}}^{N-1}$ becomes a fiber space with the orbits of $G(\mathcal{A})$ as fibers and base $U(N) / G(\mathcal{A})$. Then a goal state $\psi_{f}$ can only be reached from $\psi_{0}$ if $\psi_{0}$ and $\psi_{f}$ belong to the same fiber. The system is not controllable purely by the action of the unitary evolution $\int \exp (i \tau H(\tau)) d \tau$ but may be controllable by the joint action of measurement plus evolution in the following sense:

Theorem: Given any goal state $\psi_{f}$, there is a family of observables $M\left(\psi_{f}\right)$ such that measurement of one of these observables on any $\psi_{0}$ plus unitary evolution leads to $\psi_{f}$ if $G(\mathcal{A})$ is either $O(N)$ or $S p\left(\frac{1}{2} N\right)$.

Proof: If $G(\mathcal{A})=O(N)$ or $S p\left(\frac{1}{2} N\right)$ we may choose an orthonormal basis $\left\{\phi_{i}\right\}$ for $S^{N-1}$ in the orbit $G(\mathcal{A}) \psi_{f}$. Construct an observable $M=\sum_{i} a_{i} P_{\phi_{i}}$, $P_{\phi_{i}}$ being the projector on $\phi_{i}$. Measuring this observable on any state $\psi_{0}$ and recording the measured value $a_{k}$ the state becomes $\phi_{k}$ and then, by unitary evolution, $\psi_{f}$ may be reached.

Remarks: 
(i) Because of both the arbitrary nature of the eigenvalues $a_{i}$ and of the orthonormal basis, there is a large family of observables appropriate for this type of control.

(ii) In the result above the state $\psi_{f}$ is fixed, but $\psi_{0}$ is arbitrary. If both $\psi_{0}$ and $\psi_{f}$ are fixed a much simpler set of controlling interactions $H_{j}$ may be sufficient. $\psi_{0}$ being fixed, one constructs the $M$ observable by $N-1$ vectors in the $N$-1-dimensional subspace orthogonal to $\psi_{0}$ plus a single vector in the orbit $G(\mathcal{A}) \psi_{f}$, non-orthogonal to $\psi_{0}$. Then, $G(\mathcal{A})$ may be a much smaller subgroup than the ones in the theorem.

One sees that, if properly used, the state disturbing effects of quantum measurement, rather than being a nuisance, add controlling power over quantum processes.

\section{Examples:}

(i) A simple example of non-controllable 3-level system has been discussed by Solomon and Schirmer[13]. Let

$$
H=H_{0}+u(t) H_{1}
$$

with

$$
H_{0}=\mu\left(\begin{array}{rrr}
-1 & 0 & 0 \\
0 & 0 & 0 \\
0 & 0 & 1
\end{array}\right) ; \quad H_{1}=d\left(\begin{array}{ccc}
0 & 1 & 0 \\
1 & 0 & 1 \\
0 & 1 & 0
\end{array}\right)
$$

$H_{0}$ and $H_{1}$ generate the algebra of $S O(3)$, therefore the system is not controllable by unitary evolution. The Strocchi map evolution equations are

$$
\frac{d}{d t}\left(\begin{array}{c}
\vec{q} \\
\vec{p}
\end{array}\right)=\left(\begin{array}{rr}
0 & A \\
-A & 0
\end{array}\right)\left(\begin{array}{c}
\vec{q} \\
\vec{p}
\end{array}\right)
$$

$A$ being the matrix $\left(\begin{array}{ccc}-\mu & u(t) d & 0 \\ u(t) d & 0 & u(t) d \\ 0 & u(t) d & \mu\end{array}\right)$. Eq.(25) splits in block form

$$
\frac{d}{d t}\left(\begin{array}{l}
\overrightarrow{q+i p} \\
\overrightarrow{q-i p}
\end{array}\right)=i\left(\begin{array}{rr}
-A & 0 \\
0 & A
\end{array}\right)\left(\begin{array}{l}
\overrightarrow{q+i p} \\
\overrightarrow{q-i p}
\end{array}\right)
$$

exhibiting the $S O(3)$ nature of the control. In terms of the Hilbert space wave functions, Eq.(26) means that $\psi^{*}$ cannot be reached from $\psi$. Three 
one-parameters subgroups of the $S O(3)$ control group, to be used later on, are

$$
h_{1}(\theta):
$$

$$
\begin{aligned}
& \left(\begin{array}{c}
\vec{q} \\
\vec{p}
\end{array}\right) \\
= & \left(\begin{array}{cccccc}
\frac{1}{2}(\cos \theta+1) & 0 & \frac{1}{2}(\cos \theta-1) & 0 & \frac{-1}{\sqrt{2}} \sin \theta & 0 \\
0 & \cos \theta & 0 & \frac{-1}{\sqrt{2}} \sin \theta & 0 & \frac{-1}{\sqrt{2}} \sin \theta \\
\frac{1}{2}(\cos \theta-1) & 0 & \frac{1}{2}(\cos \theta+1) & 0 & \frac{-1}{\sqrt{2}} \sin \theta & 0 \\
0 & \frac{1}{\sqrt{2}} \sin \theta & 0 & \frac{1}{2}(\cos \theta+1) & 0 & \frac{1}{2}(\cos \theta-1) \\
\frac{1}{\sqrt{2}} \sin \theta & 0 & \frac{1}{\sqrt{2}} \sin \theta & 0 & \cos \theta & 0 \\
0 & \frac{1}{\sqrt{2}} \sin \theta & 0 & \frac{1}{2}(\cos \theta-1) & 0 & \frac{1}{2}(\cos \theta+1)
\end{array}\right)\left(\begin{array}{c}
\vec{q} \\
\vec{p}
\end{array}\right)
\end{aligned}
$$

$h_{2}(\theta):$

$$
\begin{aligned}
& \left(\begin{array}{l}
\vec{q} \\
\vec{p}
\end{array}\right) \\
& =\left(\begin{array}{cccccc}
\frac{1}{2}(\cos \theta+1) & \frac{-1}{\sqrt{2}} \sin \theta & \frac{1}{2}(1-\cos \theta) & 0 & 0 & 0 \\
\frac{1}{\sqrt{2}} \sin \theta & \cos \theta & \frac{-1}{\sqrt{2}} \sin \theta & 0 & 0 & 0 \\
\frac{1}{2}(1-\cos \theta) & \frac{1}{\sqrt{2}} \sin \theta & \frac{1}{2}(\cos \theta+1) & 0 & 0 & 0 \\
0 & 0 & 0 & \frac{1}{2}(\cos \theta+1) & \frac{-1}{\sqrt{2}} \sin \theta & \frac{1}{2}(1-\cos \theta) \\
0 & 0 & 0 & \frac{1}{\sqrt{2}} \sin \theta & \cos \theta & \frac{-1}{\sqrt{2}} \sin \theta \\
0 & 0 & 0 & \frac{1}{2}(1-\cos \theta) & \frac{1}{\sqrt{2}} \sin \theta & \frac{1}{2}(\cos \theta+1)
\end{array}\right)\left(\begin{array}{c}
\vec{q} \\
\vec{p}
\end{array}\right)
\end{aligned}
$$

$h_{3}(\theta):$

$$
\left(\begin{array}{l}
\vec{q} \\
\vec{p}
\end{array}\right)=\left(\begin{array}{cccccc}
\cos \theta & 0 & 0 & \sin \theta & 0 & 0 \\
0 & 1 & 0 & 0 & 0 & 0 \\
0 & 0 & \cos \theta & 0 & 0 & -\sin \theta \\
-\sin \theta & 0 & 0 & \cos \theta & 0 & 0 \\
0 & 0 & 0 & 0 & 1 & 0 \\
0 & 0 & \sin \theta & 0 & 0 & \cos \theta
\end{array}\right)\left(\begin{array}{c}
\vec{q} \\
\vec{p}
\end{array}\right)
$$

Although not controllable by unitary evolution alone, the system is controllable by measurement plus evolution. Consider, for example, 
$\psi_{f}=\left\{\vec{q}=(0,0,0), \vec{p}=\left(\frac{1}{\sqrt{2}}, 0, \frac{1}{\sqrt{2}}\right)\right\}$ as the goal state. By applying $h_{1}\left(-\frac{\pi}{2}\right)$ and $h_{2}\left(\frac{\pi}{2}\right) h_{1}\left(-\frac{\pi}{2}\right)$ to this state one obtains an orthogonal set

$$
\begin{aligned}
& \psi_{1}=\{\vec{q}=(0,1,0), \vec{p}=(0,0,0)\} \\
& \psi_{2}=\left\{\vec{q}=\left(\frac{-1}{\sqrt{2}}, 0, \frac{1}{\sqrt{2}}\right), \vec{p}=(0,0,0)\right\} \\
& \psi_{3}=\left\{\vec{q}=(0,0,0), \vec{p}=\left(\frac{1}{\sqrt{2}}, 0, \frac{1}{\sqrt{2}}\right)\right\}
\end{aligned}
$$

Denoting by $P_{i}$ the projectors on the states $\psi_{i}$, measurement of an arbitrary state by any one of the observables in the family

$$
\sum_{i} a_{i} P_{i}
$$

projects it on the fiber of $\psi_{f}$ and then, by unitary evolution, $\psi_{f}$ may be reached.

(ii) So far, control by measurement plus evolution has been discussed for finite-dimensional spaces. However the same technique may be used in infinite-dimensional spaces to reach a large number of states. This is illustrated for kicked motions in the torus. Let $\vec{x}=\left(x_{1}, x_{2} \in[-\pi, \pi)\right)$ be coordinates in the 2-torus $T^{2}$ and the system Hamiltonian $H$ be

$H=-\frac{\Delta}{2}+\sum_{n}\left\{\frac{-1}{2} u_{1}(t)(\vec{x} \bullet A \bullet i \nabla+i \nabla \bullet A \bullet \vec{x})+u_{2}(t) x_{1}+u_{3}(t) x_{2}\right\} \delta(t-n \tau)$

The switching functions $u_{i}(t)$ take values 0 or \pm 1 and the matrix $A$ is chosen such that $M=\exp (A)$ is a hyperbolic $2 \times 2$ matrix with integers entries and determinant one, this being the condition that insures unitarity of the Floquet operator[16]. The system is a controlled version of the configurational quantum cat[14], a system that describes a charged particle acted upon by electromagnetic pulses. When $u_{1}(t) \neq 0$ the Floquet operator has continuous spectrum and quantum chaos, in the sense of positive quantum Lyapunov exponents[15] [16]. The free and kicked components of the Floquet operator are

$$
\begin{aligned}
U_{0} & =\exp \left(i \frac{\Delta}{2} \tau\right) \\
U_{1} & =\exp \left(\frac{i}{2}(\vec{x} \bullet A \bullet i \nabla+i \nabla \bullet A \bullet \vec{x})\right) \\
U_{2} & =\exp \left(-i x_{1}\right) \\
U_{3} & =\exp \left(-i x_{2}\right)
\end{aligned}
$$


$U_{0}$ corresponds to free propagation, $U_{1}$ to the action of a linear vector field and $U_{2}, U_{3}$ to scalar potentials. The eigenstates of momentum form a numerable normalized basis

$$
|\vec{k}\rangle=\frac{1}{\sqrt{2 \pi}} e^{i \vec{k} \cdot \vec{x}} \quad \vec{k}=\left(k_{1}, k_{2}\right) \quad k_{i} \in \mathbb{Z}
$$

which is dense on the Hilbert space of the system. The kicks $U_{1}$ act on these states as follows

$$
U_{1}|\vec{k}\rangle=\left|M^{-1} \vec{k}\right\rangle
$$

By the action of kicks of type $U_{1}$ the momentum eigenstates move along hyperbolas. The kicks $U_{2}$ and $U_{3}$ move between different hyperbola. In between kicks, free propagation just changes the phase of the states.

Given now an arbitrary state $\psi$, measuring its momentum the state becomes projected on a momentum eigenstate with known eigenvalue, if the result of the measurement is recorded. By switching on the appropriate sequence of kicks $U_{i}$ it is then possible to reach any momentum eigenstate. Therefore one sees that with measurement and three controlling fields one can, from an arbitrary $\psi$, reach any state in an infinite-dimensional dense set.

\section{Nonlinear and optimal control}

Classical control is a very mature field where many useful techniques and results have been found, many of them still without parallel in quantum control. The Strocchi map, yielding a picture of quantum evolution as Hamiltonian dynamics in a classical-like phase-space, may be the appropriate tool to carry over techniques from classical to quantum control. We will give two examples:

\section{(i) Optimal control}

Optimal control is an important issue both in classical and quantum control and, in quantum control, it has been discussed using variational techniques[17] [18] [19]. However, in classical control, Pontryagin maximum principle[20] provides a more general framework in the sense that it does not require differentiability and can handle piecewise continuous and magnitudelimited control. We now show how to carry over this principle to quantum control using the Strocchi map. 
In addition to the $2 N$ phase-space variables $\left(x_{k}=q_{k} ; x_{k+N}=p_{k}\right)$ with dynamical laws

$$
\begin{aligned}
& \frac{d}{d t} x_{k}=\frac{d}{d t} q_{k}=\frac{\partial}{\partial p_{k}} \mathbb{H}=X_{k} \\
& \frac{d}{d t} x_{k+N}=\frac{d}{d t} p_{k}=-\frac{\partial}{\partial q_{k}} \mathbb{H}=X_{k+N}
\end{aligned}
$$

obtained from (7), we introduce a variable $x_{0}$ with dynamical law

$$
\frac{d}{d t} x_{0}=X_{0}(x, u, t)
$$

with

$$
F=\int_{0}^{T} X_{0}(x, u, t) d t
$$

being the performance functional to be minimized. If, for example, minimal controlling energy is desired $X_{0}(x, u, t)=|u(t)|^{2}$, etc.

Then, for each variable in the set $\vec{x}=\left(x_{0}, q_{1}, \cdots, q_{N}, p_{1}, \cdots, p_{N}\right)$ an adjoint variable $\phi_{i}$ is defined and a new "Hamiltonian" $\mathbf{H}(x, \phi, u)$

$$
\mathbf{H}(x, \phi, u)=\sum_{i=0}^{2 N} \phi_{i} X_{i}(x, u)
$$

For each specified initial $\left(x_{k}=q_{k}=q_{k}(0), x_{k+N}=p_{k}=p_{k}(0)\right)$ and final state $\left(x_{k}=q_{k}=q_{k}(T), x_{k+N}=p_{k}=p_{k}(T)\right)$, the optimal control that, in time $T$, minimizes the functional $F$ is obtained by integration of

$$
\begin{aligned}
\frac{d}{d t} x_{k} & =\frac{\partial}{\partial \phi_{k}} \mathbf{H}(x, \phi, u) \\
\frac{d}{d t} \phi_{k} & =-\frac{\partial}{\partial x_{k}} \mathbf{H}(x, \phi, u) \\
\vec{u} & =\arg \max \vec{u} \in \Omega
\end{aligned}
$$

$\Omega$ being the domain of allowed controls.

In the variational formulation of optimal quantum control, the technique that has been used is to minimize the functional

$J=\langle\psi(T)|(1-\Pi)| \psi(T)\rangle+\int_{0}^{T} X_{0}(\psi, u, t) d t+\int_{0}^{T} \operatorname{Im}\left\langle\zeta(t)\left|i \partial_{t}-H\right| \psi(t)\right\rangle$

$\Pi$ being the projector onto the target state. The first two equations in (40) correspond to the Schrödinger equation and to the equation for the Lagrange 
multiplier $\zeta(t)$, whereas the third one corresponds to the equation obtained by variation $\delta \vec{u}$ of the control parameters. However the set (40) is more general in that it does not require differentiability in $u$ of $J$ and allows the specification of arbitrary control domains $\Omega$.

\section{(ii) Sliding mode techniques in quantum control}

A very robust tool used in classical control is the technique of variable structure control leading to sliding modes[21]. The design of a variable structure control has two steps. First, a switching surface must be chosen so that the dynamical system restricted to the surface has the desired dynamics. Second, a switched control must be found to drive the system to the switching surface and, upon interception, to maintain it there. For this step a Lyapunov function approach is used, the gradient of the Lyapunov function being negative in the neighborhood of the switching surface. In this way the tangent vectors to the state trajectory point towards the surface. The system is attracted to the switching surface and, once having intercepted it, remains there for all subsequent times. Then, the state trajectory is said to be a sliding mode.

From the very nature of the technique one sees that, at least close to the switching surface, the dynamics must have a dissipative component and therefore a purely Hamiltonian control cannot be used. However, as seen in Section 1, observation introduces non-Hamiltonian effects which might play for quantum dynamics the same role as dissipation in classical control.

Consider again the non-controllable example of the previous section and suppose that one wants to stabilize the middle energy level. Starting from an arbitrary initial state one measures the energy. If the result is zero we are done because it means that the state was projected on the middle level. Otherwise if the result is $+\mu$ or $-\mu$, one knows from the controlling subgroups in Eqs.(27-29), that there is a control that changes the upper or the lower level into a state with a $50 \%$ probability of being projected on the middle energy state by an energy measurement. One performs this control plus measurement operation until the result of the measurement is zero. Afterwards the control is switched off. If there are no disturbances the state remains in the middle level. Otherwise if there are some decoherent interaction with the environment, the state should be periodically measured. If the disturbance is small or if the intervals between measurements are small, there is a

high probability that the system will be projected back on the middle level, 
without any need for further controlling operations.

\section{Conclusions}

In addition to a formulation of quantum control in a symplectic geometry setting, the main result of this work is the proposal of a new protocol for quantum control, which we have called "control by measurement plus evolution". It extends the scope of quantum controllability and is applicable both to finite and infinite-dimensional level systems.

Different aspects of quantum control are united in the framework of the Strocchi map, allowing new insights in optimal control and nonlinear control techniques. We think that the potential of this picture is not exhausted and more analogies with classical problems may be used to obtain progress in the quantum domain.

\section{References}

[1] F. Strocchi; Rev. Mod. Phys. 38 (1966) 36.

[2] T. W. B. Kibble; Commun. Math. Phys. 65 (1979) 189.

[3] A. Heslot; Phys. Rev. D31 (1985) 1341.

[4] J. Anadan and Y. Aharonov; Phys. Rev. Lett. 65 (1990) 1697.

[5] R. Cirelli, A. Manià and L. Pizzocchero; J. Math. Phys. 31 (1990) 2891.

[6] A. Ashtekar and T. A. Schilling; in On Einstein's path. Essays in honor of Engelbert Schucking, Alex Harvey (Ed.), Springer, N. Y. 1999, pages 23-65.

[7] V. I. Man'ko and G. Marmo; Phys. Scr. 60 (1999) 111.

[8] G. Marmo, G. Morandi, A. Simoni, and F. Ventriglia; J. Phys. A 35 (2002) 8393.

[9] C. M. Caves and G. J. Milburn; Phys. Rev. A 36 (1987) 5543. 
[10] G. M. Huang, T. J. Tarn and J. W. Clark; J. Math. Phys. 24 (1983) 2608.

[11] H. Fu, S. G. Schirmer and I. S. Solomon; J. Phys. A : Math. Gen. 34 (2001) 1679.

[12] V. Ramakrishna, M. V. Salapaka, M. Dahleh, H. Rabitz and A. Peirce; Phys. Rev. A51 (1995) 960.

[13] A. I. Solomon and S. G. Schirmer; quant-ph/0110030

[14] S. Weigert; Z. Phys. B 80 (1990) 3; Phys. Rev. A48 (1993) 1780.

[15] V. I. Man'ko and R. Vilela Mendes; Physica D 145 (2000) 330.

[16] V. I. Man'ko and R. Vilela Mendes; Phys. Lett. A 300 (2002) 353.

[17] M. Dahleh, A. P. Pierce and H. Rabitz; Phys. Rev. A42 (1990) 1065.

[18] M. Demiralp and H. Rabitz; Phys. Rev. A47 (1993) 809; A47 (1993) 831.

[19] C. D. Schwieters and H. Rabitz; Phys. Rev. A48 (1993) 2549.

[20] L. S. Pontryagin, V. G. Boltianski, R. V. Gamkrelidze and E. F. Mishchenko; The Mathematical Theory of Optimal Processes, Interscience Publishers, New York 1962.

[21] R. A. DeCarlo, S. H. Zak and G. P. Matthews; Proc. IEEE 76 (1988) 212. 